\section{Polymeric nanocomposites coatings for the corrosion protection of steel}

\section{Rana Magdy ,Anfal Alselity , Dr. Abdul Shakoor}

\section{Introduction}

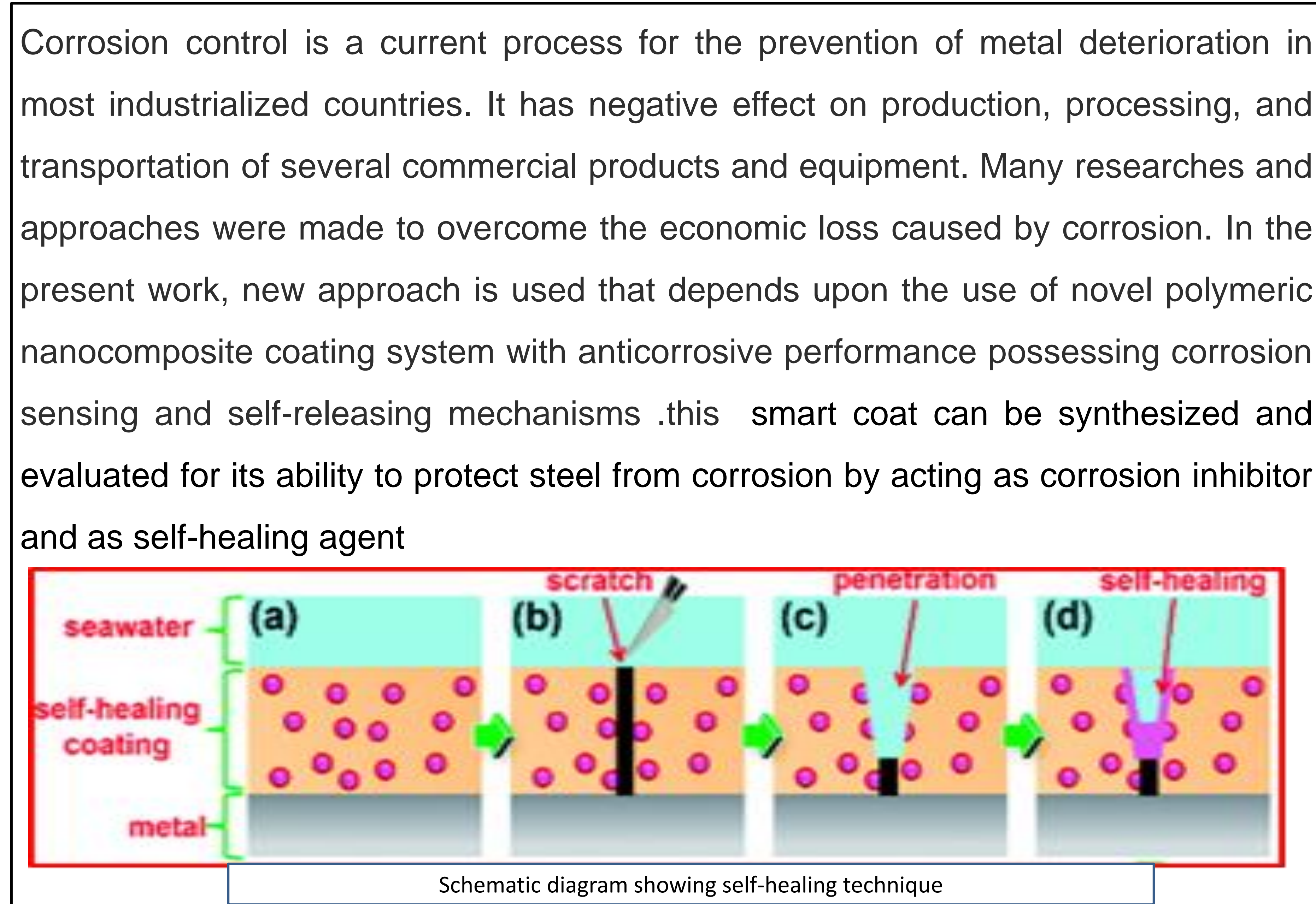

\section{Results and discussion}

1. Scanning electron microscope: SEM results showing the smooth structure of the nano containers at different scales that ensures loading With the MTU

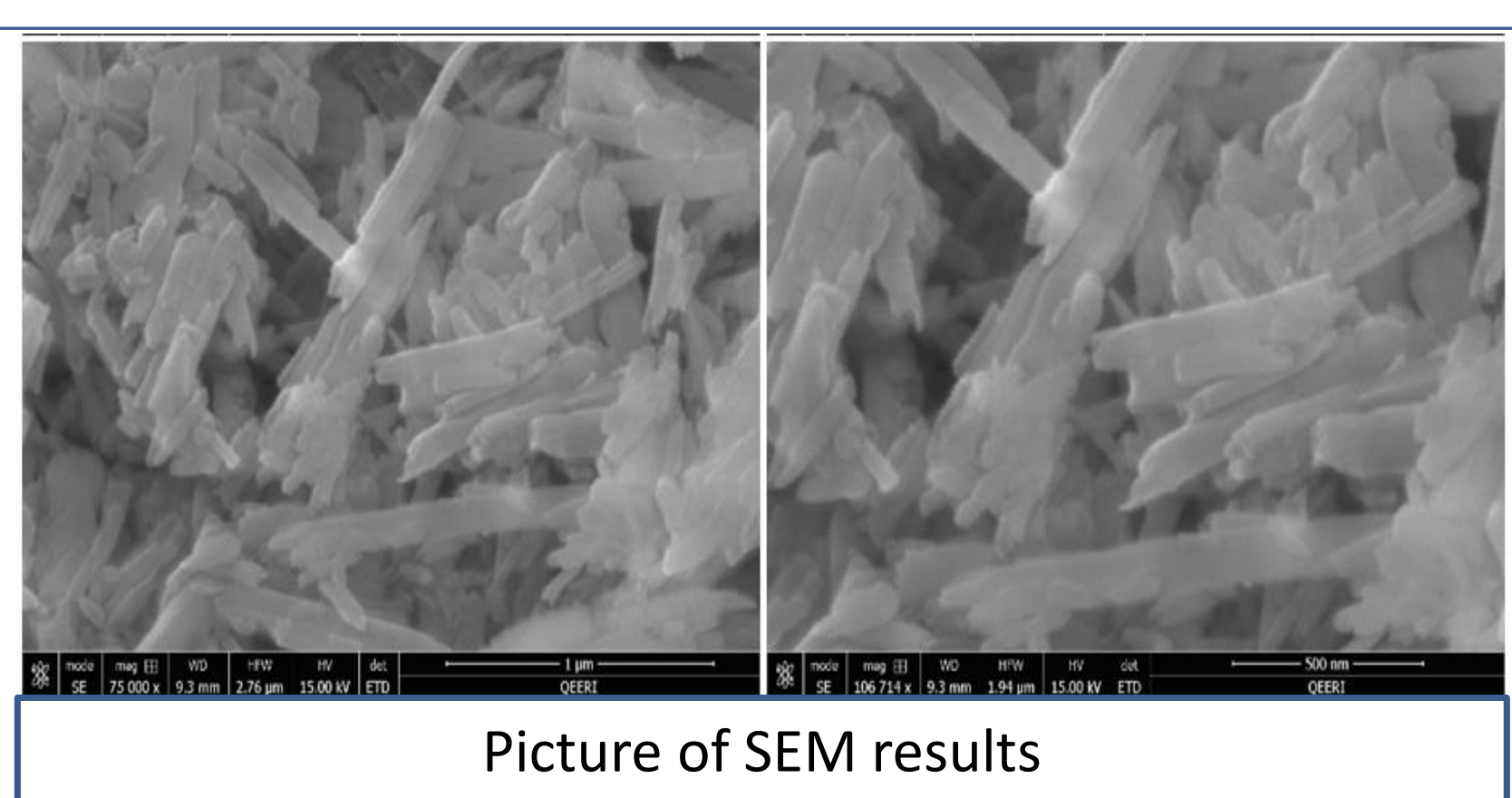

2. FTIR ( FOURIER TRANSFORM INFRARED SPECTROSCOPY):

The change in the characteristic peaks observed for loaded product that indicates the loading of the inhibitor in HNTs.

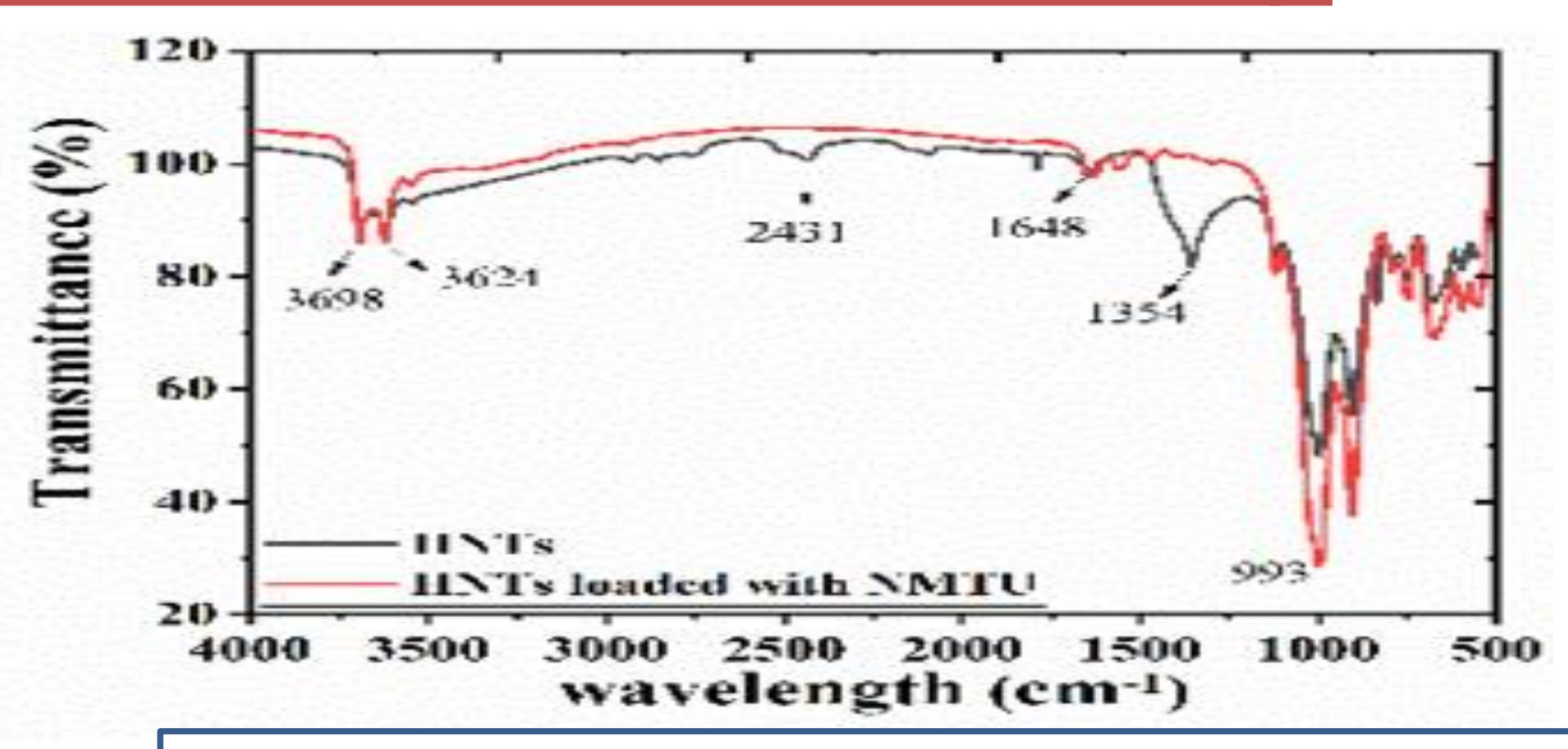

\section{XRD (X-RAY DIFFRACTION DEVICE)}

- the black line is nanotubes alone

-The red line show that nanotubes we

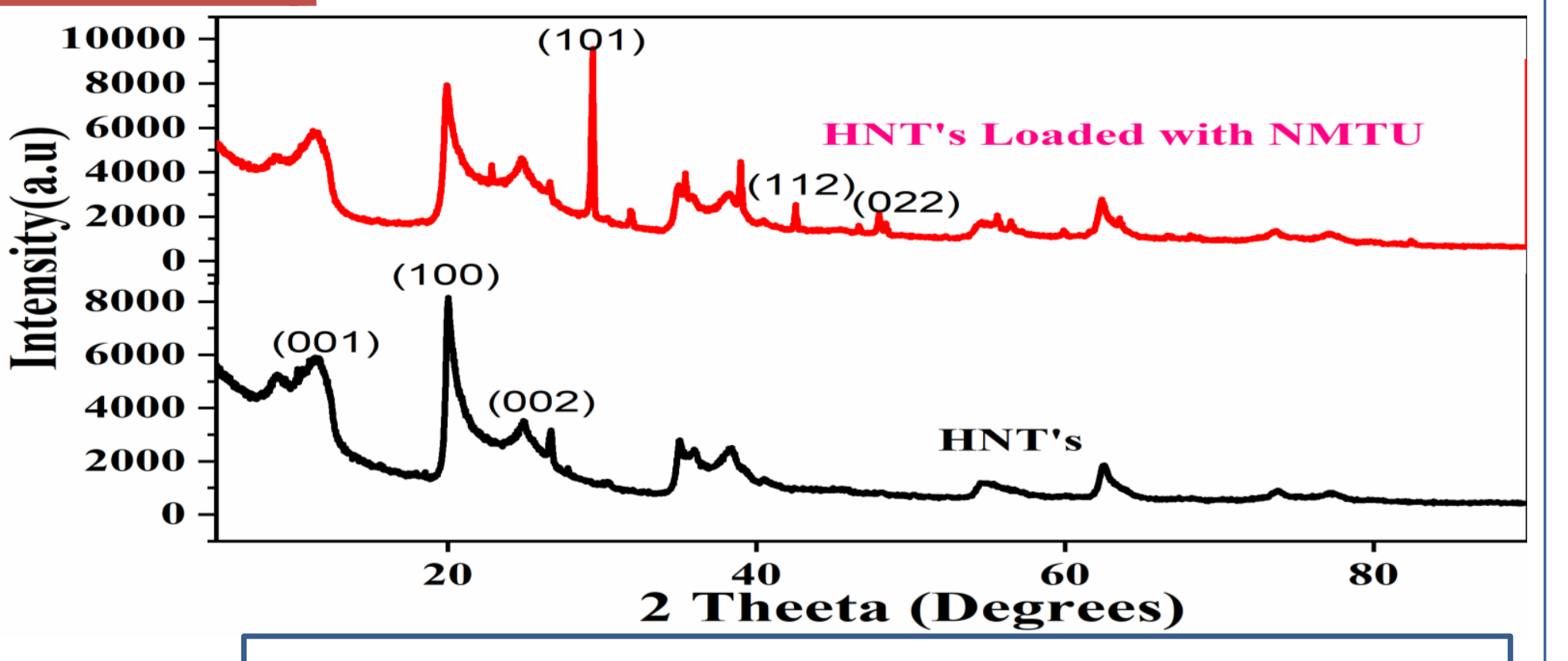

\section{TGA (thermogravimetric analyzer):}

The initial weight loss till $100{ }^{\circ} \mathrm{C}$ is due to moisture content. Around $5 \%$ product of NMTU is successfully loaded inside HNTs

\section{-3. Gamry 3300:}

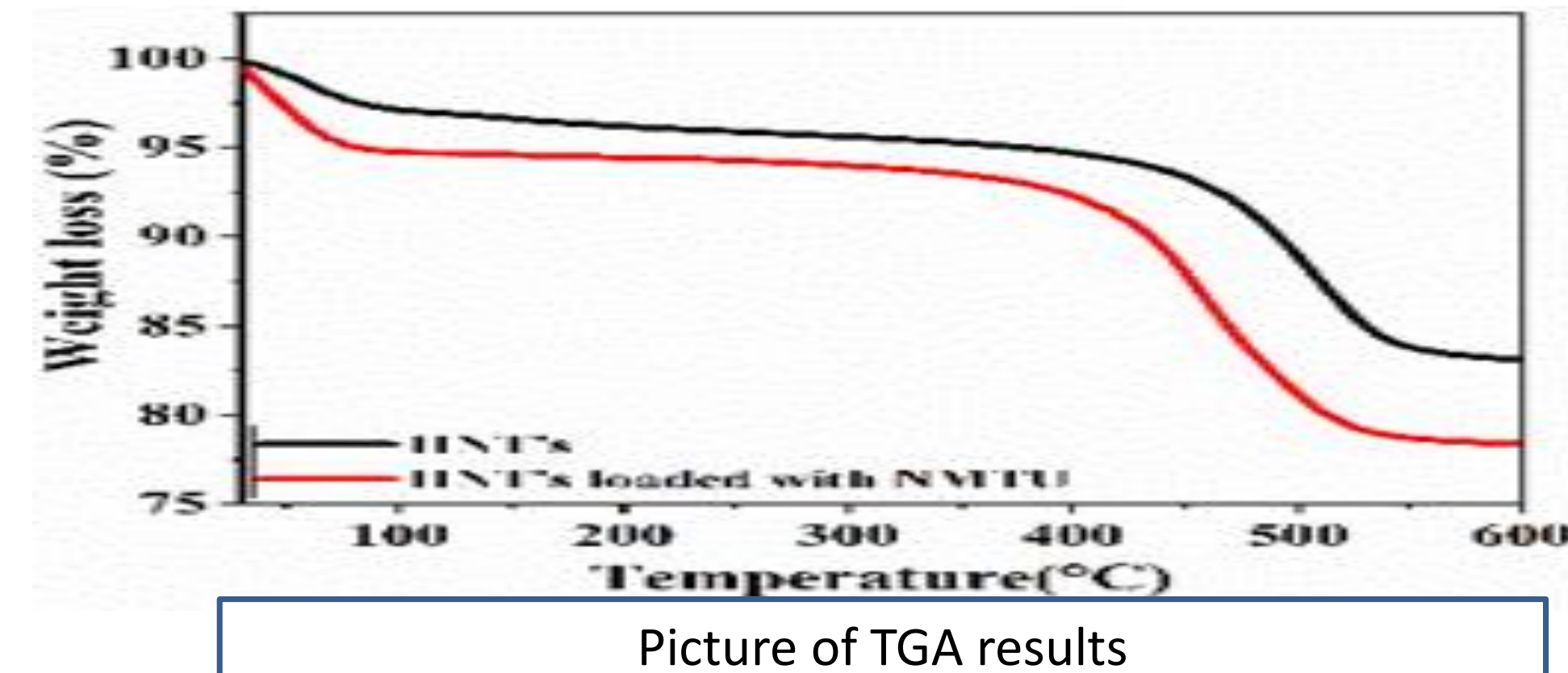

The EIS results showed the corrosion resistance of reference coatings decreases with time that can be shown in bode and phase angle plot The EIS results showed the corrosion resistance of modified coating increases with time that can be shown in bode and phase angle plot
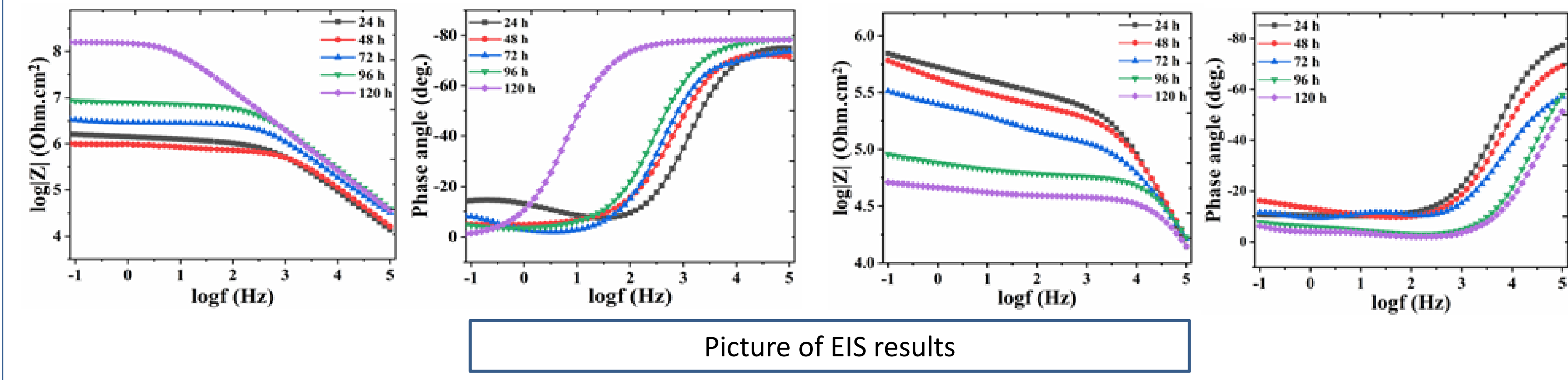

\section{Experimental Steps}

The procedure included 3 processes:

\section{Loading process}

NMTU + NANOTUBES = LOADED PRODUCT

a. Mixing $2 \mathrm{gm}$ of MTU and $1 \mathrm{gm}$ of nanotubes with $10 \mathrm{ml}$ water and stirring them by magnetic stirrer

a. Placing the sample in the vacuum chamber to help encapsulation of MTU into nanotubes for 24 hours

a. Centrifuge for $15 \mathrm{~min}$. using centrifuge device to separate the loaded product b. Drying the sample in room temperature for 24 hours

\section{2. coating process}

a. Polishing of the steel substrate using polisher device

b. Mix 10 gram pf epoxy and 25gram hardener and1.25 gram loaded product

C. Removing bubbles in the sample Using ultrasonic bath D. Coating the steel substrate with the sample

\section{Testing process}

a. Structural characterization using SEM, FTIR, TGA and XRD

b. Corrosion testing using GAMRY 3300 device to measure the corrosion resistance

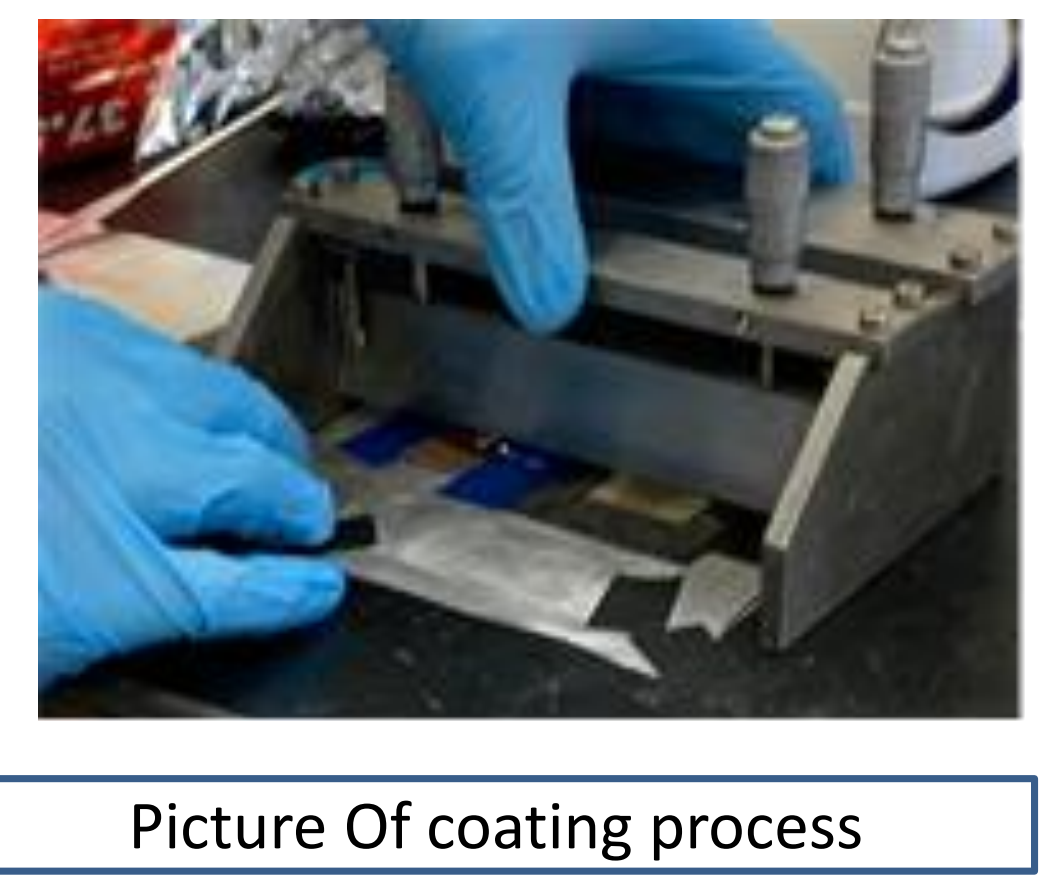

(n)

\section{Conclusions Recommendations}

The encapsulated active material (NMTU) was released gradually from the nanotubes after the scratch was formed, which begin to self-heal and resist the corrosive environment (as shown in EIS results)

This ensures the ability of NMTU to perform corrosion inhibition and self-healing on the surface of steel substrate, protecting it from corrosion. This consequently emphasizes the research hypothesis that the new nanocomposite smart coating is able to protect the steel from corrosion.

future researches for the development of new loaded inhibitors nano containers of higher performance and more environmentally friendly is highly recommended. Also it is recommended to try these smart coatings in different applications in(Oil and gas, Automotive, Aerospace, Textile, Manufacturing, Electrical fields) and further studies could be made under various corrosive conditions.

Scientific advances for performance assessment can result in an increase in reliability and safety, reduction in costs, and conservation of materials and energy

\section{Acknowledgment}

This research is supported, and under the supervision of Al-Bairaq project, Center for Advanced Materials (CAM), Qatar University.

We are immensely grateful to Dr. Abd Shakour, for all the insight, expertise and support he has provided in order to execute this research.

We thank Dr. Noora Al-Thani, Director of AL-Bairaq project and Head of outreach and resource, Center of Advanced Materials (CAM), Qatar University.

\section{References}

- 1 Vijayan, P., \& Al-Maadeed, M. (2019). Self-Repairing Composites for Corrosion Protection: A Review on Recent Strategies and Evaluation 